\title{
Erythroblastic sarcoma transformation from a chronic myeloid neoplasm with FGFR1 rearrangement presenting as a pleural effusion: a case report
}

\author{
Joanna C. Dalland ${ }^{1}$ (D) - Alexandra Higgins ${ }^{2} \cdot$ Horatiu Olteanu ${ }^{1} \cdot$ Kaaren K. Reichard ${ }^{1} \cdot$ Reid Meyer $^{3}$. \\ Gregory E. Otteson ${ }^{1} \cdot$ Patricia T. Greipp ${ }^{3} \cdot$ Rebecca L. King ${ }^{1}$
}

Received: 23 November 2020 / Accepted: 14 December 2020 / Published online: 3 January 2021

(C) The Author(s), under exclusive licence to Springer-Verlag GmbH, DE part of Springer Nature 2021

\begin{abstract}
Myeloid/lymphoid neoplasms with FGFR1 rearrangement (MLN-FGFR1) comprise a heterogeneous group of neoplasms that can present as a myeloproliferative neoplasm, a myelodysplastic/myeloproliferative neoplasm, acute myeloid leukemia, T or B lymphoblastic leukemia/lymphoma, or mixed phenotype acute leukemia. The overall outcome of these neoplasms is poor, and these patients often show cytogenetic evolution with gain of additional chromosomal abnormalities leading to transformation to acute leukemia. Pure erythroid leukemia and erythroblastic sarcoma evolving from a preexisting chronic myeloid neoplasm have rarely been described; however, this has not been reported in MLN-FGFR1. Herein we describe a biopsy-proven chronic myeloid neoplasm with FGFR1 rearrangement with transformation to erythroblastic sarcoma presenting as a pleural effusion.
\end{abstract}

Keywords FGFR1 rearrangement · Erythroblastic sarcoma · Pure erythroid leukemia · Running title: Erythroblastic sarcoma transformation from chronic myeloid neoplasm with FGFR1 rearrangement.

\section{Introduction}

Myeloid/lymphoid neoplasms with FGFR1 rearrangement (MLN-FGFR1) comprise a heterogeneous group of WHOdefined neoplasms associated with a $\mathrm{t}(8 ; 13)(\mathrm{p} 11.2 ; \mathrm{q} 12)$ or a variant translocation leading to FGFR1 rearrangement [1]. As the rearrangement occurs in a pluripotent stem cell, the presentation can be as a myeloproliferative neoplasm (MPN), myelodysplastic/myeloproliferative neoplasm (MDS/MPN), acute myeloid leukemia, or lymphoblastic leukemia. Regardless of initial presentation, these neoplasms are

Joanna C. Dalland

dalland.joanna@mayo.edu

1 Department of Laboratory Medicine and Pathology, Division of Hematopathology, Mayo Clinic, 200 First St. SW Hilton Building 7, Rochester, MN 55905, USA

2 Department of Internal Medicine, Division of Hematology, Mayo Clinic, Rochester, MN, USA

3 Department of Laboratory Medicine and Pathology, Division of Laboratory Genetics and Genomics, Mayo Clinic, Rochester, MN, USA associated with a poor prognosis due to high incidence of transformation to acute leukemia $[1,2]$.

Pure erythroid leukemia (PEL) is a rare type of acute leukemia composed of immature erythroid lineage cells with no evidence of a significant myeloblast component [1]. It is frequently associated with a complex karyotype and an aggressive clinical course [3]. Erythroblastic sarcoma (EBS) is composed of immature erythroid lineage cells presenting in an extramedullary site and is similarly associated with a poor prognosis $[1,4]$. We report the first case of a chronic MLN$F G F R 1$ with transformation to EBS.

\section{Clinical history}

A 91-year-old male with history of coronary artery disease, abdominal aortic aneurysm, and obstructive sleep apnea presented for evaluation of a persistent leukocytosis found incidentally during a pre-operative evaluation. His white blood cell count (WBC) ranged from 12 to $19 \times 10^{9} / \mathrm{L}$ with the differential count notable for $46 \%$ neutrophils, $18 \%$ lymphocytes, $14 \%$ monocytes, $4 \%$ eosinophils, $15 \%$ myelocytes, and $3 \%$ metamyelocytes. This was associated with mild 
thrombocytopenia of $120 \times 10^{9} / \mathrm{L}$ and normal hemoglobin of $14.8 \mathrm{~g} / \mathrm{dL}$. Splenomegaly measuring $16 \mathrm{~cm}$ was noted on imaging. Bone marrow aspirate and biopsy (details below) showed features initially concerning for chronic myelomonocytic leukemia; however, cytogenetic studies revealed a balanced $\mathrm{t}(8 ; 13)(\mathrm{p} 11.2 ; \mathrm{q} 12)$ translocation in 19 of 20 metaphases and fluorescence in situ hybridization (FISH) studies confirmed an FGFR1 rearrangement, meeting diagnostic criteria for MLN-FGFR1. The patient was lost to follow-up and did not receive any therapy.

He presented 4 months later with new onset of dyspnea and a CT scan revealed a large left pleural effusion. At the time of admission, a complete blood cell count showed leukocytosis of $33.7 \times 10^{9} / \mathrm{L}$, mild thrombocytopenia of $101 \times 10^{9} / \mathrm{L}$, and a normal hemoglobin of $14.5 \mathrm{~g} / \mathrm{dL}$. The WBC differential count showed $70 \%$ neutrophils, $20 \%$ lymphocytes, $4 \%$ monocytes, $4 \%$ eosinophils, $1 \%$ metamyelocytes, and $1 \%$ myelocytes. Pleurocentesis was performed for diagnosis and bone marrow studies were repeated.

Following the diagnosis of EBS in the pleural fluid, the patient was started on low dose ponatinib $15 \mathrm{mg}$ daily. The pleural effusion was drained and did not recur after starting ponatinib. There was evidence of peripheral hematologic response with WBC normalization to $7.6 \times 10^{9} / \mathrm{L}$ within 6 weeks. Neutropenia developed 9 weeks after initiation of ponatinib at which time the medication was held. In the setting of neutropenic fever, functional decline, and weakness, the patient elected to transition to supportive care only and passed away 7 months after the original diagnosis of MLN-FGFR1.

\section{Materials and methods}

Wright-Giemsa stained pleural fluid cytospin and bone marrow aspirate preparations, and hematoxylin and eosin (H\&E) stained bone marrow core biopsy and cell block sections of the pleural fluid were reviewed.

A portion of the pleural fluid was submitted for flow cytometric analysis. The sample was processed by ACK lyse/wash before following standard surface and cytoplasmic staining protocols. Flow cytometry standard (FCS) data was acquired on BD FACSCanto II and BD FACSLyric cytometers. Flow cytometry data was analyzed in Beckman Coulter Kaluza, version 2.1. Images from flow cytometry data were generated with Cytognos Infinicyt, version 2.0. Antibody combinations used for flow cytometric immunophenotyping are documented in the results below.

All immunohistochemistry (IHC) was performed on 4um formalin-fixed paraffin embedded (FFPE) sections using an automated IHC platform (Benchmark XT; Ventana Medical Systems, Tucson, AZ, USA). Antibodies performed are documented in the results below.

Conventional chromosome studies [5] were performed according to standard unstimulated bone marrow culturing and GTL-banded metaphase preparation methods with trypsin and Lesihman staining. Slide preparations were digitally scanned and metaphases were karyotyped by a technologist using CytoVision software (Leica, Buffalo Grove, IL).

Interphase fluorescence in situ hybridization (FISH) for FGFR1 (8p11.2) rearrangement was performed on the pleural fluid and bone marrow aspirate following standard FISH pretreatment, hybridization, and fluorescence microscopy protocols [6] utilizing a laboratory-developed FGFR1 dual-color break-apart (BAP) strategy probe with 3'telomeric region labeled in SpectrumGreen (Abbott, Abbott Park, IL) and 5'centromeric region labeled in SpectrumOrange (Abbott, Abbott Park, IL). Two technologists scored 200 interphase nuclei using CytoVision software (Leica, Buffalo Grove, IL).

\section{Results}

Cytospin preparations and H\&E-stained cell block sections of the pleural fluid showed a discohesive population of large cells with immature chromatin, large round nuclei, multiple prominent nucleoli, and abundant deeply basophilic cytoplasm with vacuoles (Fig. 1a, b). No myeloid or erythroid maturation was seen in the background.

Flow cytometric immunophenotyping revealed a cell population accounting for $85 \%$ of total analyzed events that expressed CD45 (dim) and CD36 (bright) (Fig. 2a, b). The cells were large with increased
Fig. 1 Pleural fluid cytology. a Cytospin preparations show large immature-appearing cells with round nuclei, multiple prominent nucleoli, and abundant deeply basophilic cytoplasm with vacuoles (Wright-Giemsa, 100X oil immersion magnification). b Cell block (H\&E, 60X oil immersion magnification)

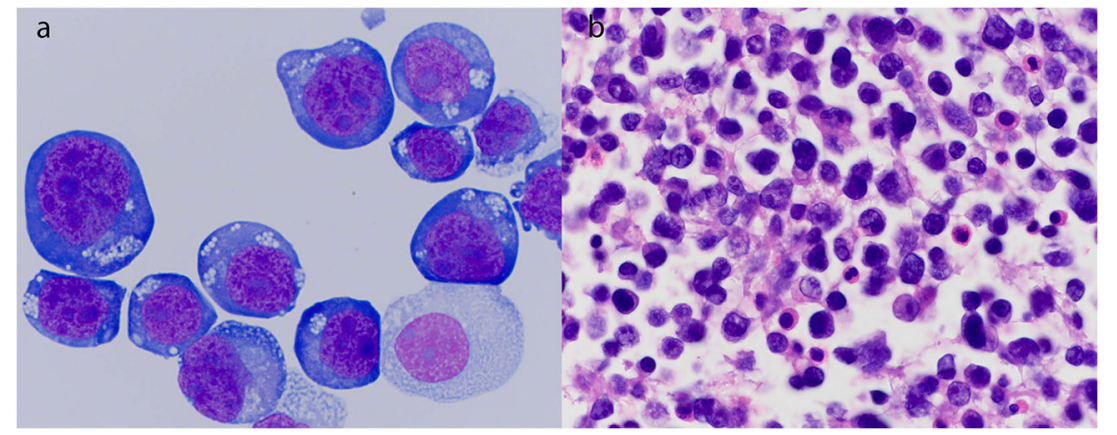


cytoplasmic complexity (vacuoles) based on forward and side angle light scatter properties. These cells were negative for CD34, CD19, CD10, CD3, CD13, CD15, CD16, CD33, CD117, HLA-DR, CD2, CD7, CD56, CD64, CD38, cMPO, nTdT, cCD22, cCD79a, and cCD3.
Immunohistochemistry showed positive staining for CD71 (diffuse/bright), CD43, and focal staining for E-cadherin (Figs. 2c-e). Rare glycophorin A and hemoglobin-positive cells were seen (Figs. 2f, g). The cells were negative for CD3, CD20, CD30, CD45, CD61, CD123, CD138, EBER-
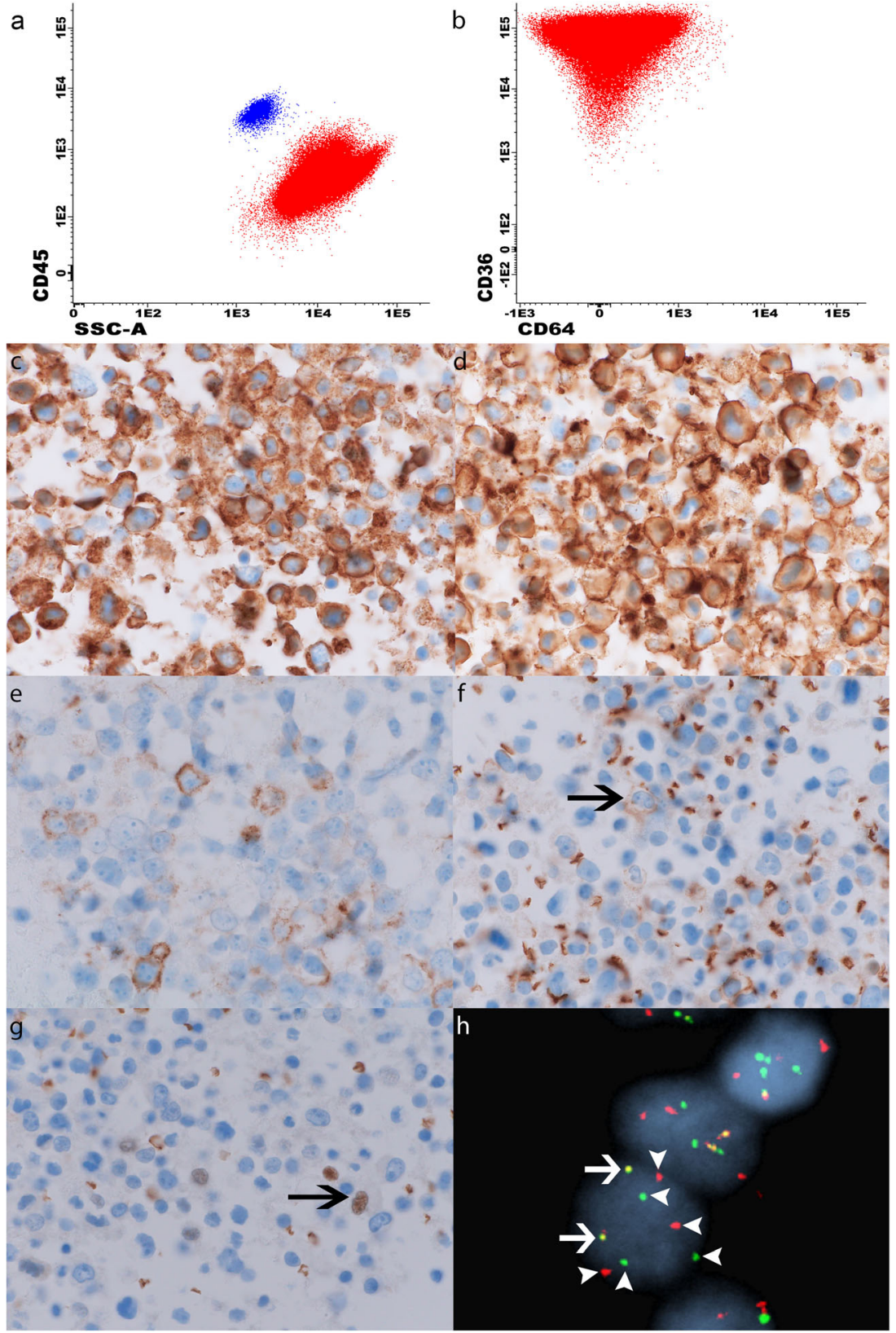

Fig. 2 Pleural fluid. a Flow cytometry CD45/SSC. The blast population (red) shows dim CD45 expression compared to the lymphocytes (blue) and exhibits increased cytoplasmic complexity (vacuoles) on SSC. b Flow cytometry CD36/CD64. The blast population (red) is positive for CD36 and is negative for CD64. c-d The blasts express CD71 (c) and CD43 (d) (60X oil immersion magnification). e A subset of the blasts is positive for E-Cadherin (60X oil immersion magnification). f-g Rare glycophorin A (f) and hemoglobin (g) positive cells are seen as indicated by the black arrows (60X oil immersion magnification). h FGFRl (8p11.2) rearrangement as demonstrated by break-apart probe FISH performed on tumor cells from a cytospin slide of the pleural fluid. The intact FGFRI gene region is represented by the yellow fusion signal (white arrows), and the disruption of the FGFRl gene region is represented by the widely separated green signal ( $\left.3^{\prime} F G F R l\right)$ and red signal $\left(5^{\prime} F G F R I\right)$ (white arrowheads). This image depicts polysomy 8 detected in $80 \%$ of nuclei with 2 yellow fusion signals and 3 widely separated 3 ' green and $5^{\prime}$ red signals, each. A near-tetraploid subclone was identified in $20 \%$ of nuclei with a similar rearrangement pattern (not shown) 
in situ hybridization, factor VIII, HHV8, AE1/AE3, OSCAR, CD117, lysozyme, myeloperoxidase, mucin, MUM1, P53, S100, SOX10, and TTF-1.

FISH showed a disruption of the FGFR1 gene region in $80 \%$ of nuclei (Fig. $2 \mathrm{~h}$ ). An additional near-tetraploid subclone was observed in $20 \%$ of nuclei. Although the morphology and unusual presentation as an isolated pleural effusion raised a broad differential diagnosis including solid tumors and lymphoma, the findings supported a transformation of the patient's chronic myeloid neoplasm with FGFR1 rearrangement, best classified as EBS.

A concurrent bone marrow biopsy was reviewed and compared with the initial diagnostic marrow. The peripheral blood showed persistent leukocytosis $\left(31.1 \times 10^{9} / \mathrm{L}\right)$, persistent mild thrombocytopenia $\left(108 \times 10^{9} / \mathrm{L}\right)$, and mild anemia $(12.8 \mathrm{~g} / \mathrm{dL})$. The WBC differential count revealed left-shifted granulocytes (3\% metamyelocytes, $13 \%$ myelocytes), neutrophilia (65\%), eosinophilia (4\%), basophilia (1\%), and monocytosis (7\%) (Fig. 3a). No circulating blasts or cells resembling those identified in the pleural fluid were seen. The bone marrow aspirate and biopsy showed a hypercellular marrow with left-shifted granulopoiesis, no increase in blasts, and frequent small hypolobated and osteoclast-like megakaryocytes (Figs. 3b-d).
Erythroid precursors showed full maturation, and no population of pronormoblastic cells, similar to those seen in the pleural fluid, was identified. Chromosome analysis showed a balanced $\mathrm{t}(8 ; 13)(\mathrm{p} 11.2 ; \mathrm{q} 12)$ translocation in all 20 metaphases, and FISH studies confirmed an FGFR1 rearrangement (Fig. 3e), supporting persistent involvement by the patient's previously diagnosed chronic myeloid neoplasm with FGFR1 rearrangement, without evidence of transformation.

\section{Discussion}

Herein we report the first case of a myeloid/lymphoid neoplasm with FGFR1 rearrangement transforming to erythroblastic sarcoma, presenting as a pleural effusion. MLN$F G F R 1$ is a pleuripotent stem cell disease that can present as a heterogeneous number of diseases. Cases that present in chronic phase typically show peripheral blood eosinophilia and can be associated with neutrophilia and monocytosis [2]. In cases presenting as acute leukemia, the blasts can be of lymphoid, myeloid, or ambiguous lineage [2]; however, to our knowledge, pure erythroid leukemia/erythroblastic sarcoma has not been previously reported.
Fig. 3 Concurrent bone marrow biopsy. a The peripheral blood shows leukocytosis with leftshifted granulocytes, neutrophilia, eosinophilia, and monocytosis (Wright-Giemsa, 60X oil immersion magnification). b-c The bone marrow aspirate shows leftshifted granulopoiesis (b) and small hypolobated megakaryocytes (c) (Wright-Giemsa, 60X oil immersion magnification, each). d The bone marrow biopsy is hypercellular (H\&E, 20X magnification). e FGFR1 (8p11.2) rearrangement as demonstrated by break-apart probe FISH performed on bone marrow aspirate cells. An intact FGFRI gene region is represented by the yellow fusion signal (white arrows), and a disruption of the FGFR1 gene region on the other chromosome 8 is represented by the widely separated green signal ( $\left.3^{\prime} F G F R 1\right)$ and red signal $\left(5^{\prime} F G F R 1\right)$ (white arrowheads)

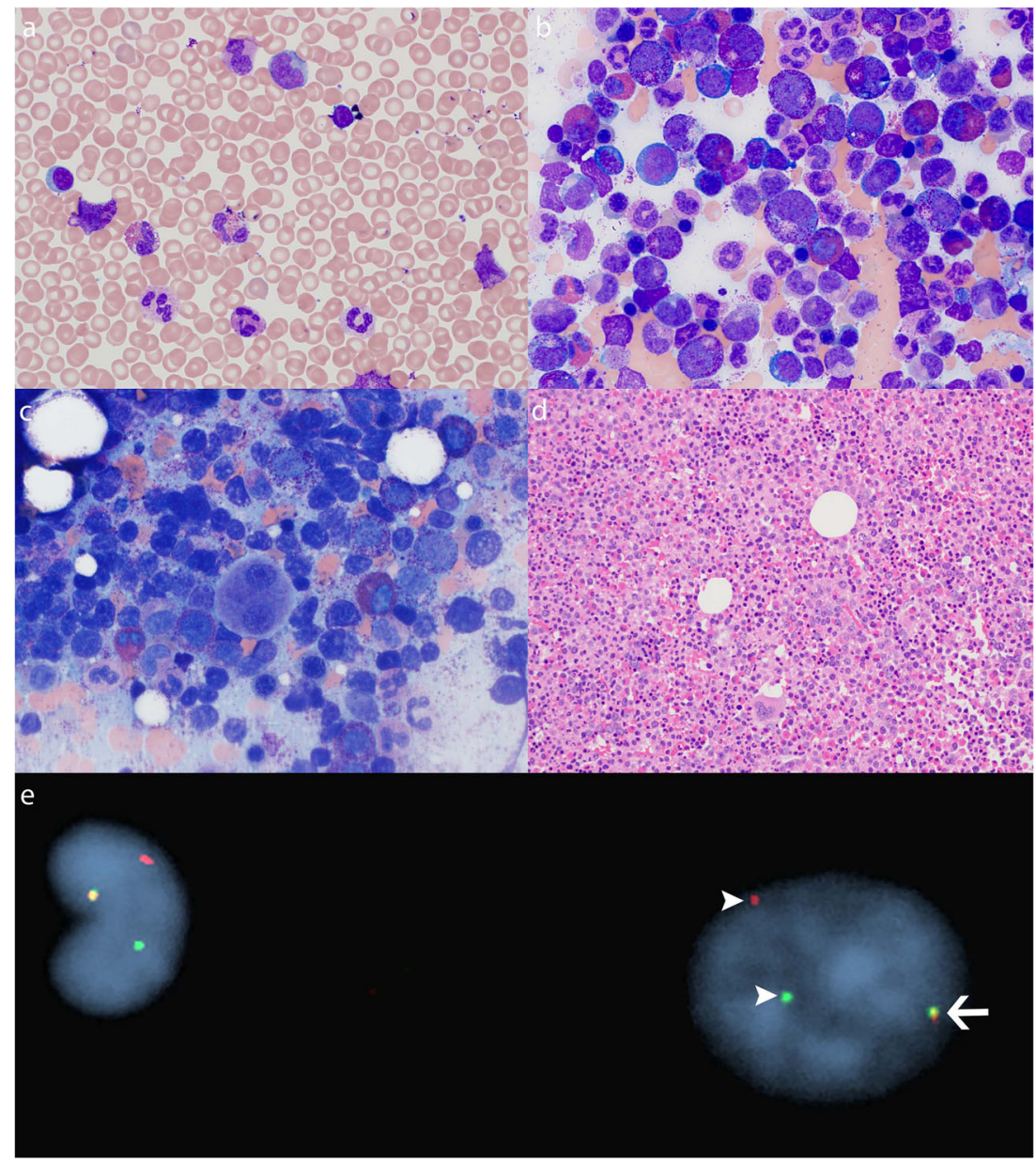


The most common translocation in MLN-FGFR1, accounting for approximately $50 \%$ of cases, is $\mathrm{t}(8 ; 13)(\mathrm{p} 11 ; \mathrm{q} 12)$ leading to fusion of the FGFR1 gene to ZMYM2 (also previously known as ZNF198) [7, 8]. Chromosome analysis in our patient revealed this common translocation. The patient's initial bone marrow biopsy showed morphologic features of a MPN, which is the typical presentation seen with this translocation [9]. It can also present as a T-lymphoblastic lymphoma [9] and is often associated with lymphadenopathy [10], neither of which were seen in our patient. Cytogenetic evolution with the gain of additional cytogenetic abnormalities in addition to $\mathrm{t}(8 ; 13)(\mathrm{p} 11 ; \mathrm{q} 12)$ can lead to disease progression and transformation [11, 12].

Pure erythroid leukemia and erythroblastic sarcoma evolving from pre-existing chronic myeloid neoplasms (CMN) is extremely rare [4, 13-15]. The largest series described 14 cases of PEL, 3 of which presented as EBS, evolving from preexisting MDS (6 PEL, 2 EBS) or a MPN (5 PEL, 1 EBS) [4]. In this series, patients with progression from a pre-existing MDS showed a shorter interval of progression. Compared with the time of initial CMN diagnosis, the PEL cases showed a more complex karyotype with a large number of clonal numeric and structural abnormalities, with no difference in complexity between those that arose from MDS versus MPN [4].

A single case report of erythroblastic sarcoma presenting as a pleural effusion arising in a patient with history of MDS/ MPN was recently reported [16]. In contrast to this recent case report, our patient presented with an isolated pleural effusion without a distinct mass identified by imaging studies. Despite the lack of a mass, we felt this was best classified as erythroblastic sarcoma given the proliferation of immature erythroid lineage cells presenting in an extramedullary site without bone marrow involvement. Erythroblastic sarcoma arising in a myeloid/lymphoid neoplasm with PDGFRA rearrangement has also rarely been reported [17]. Clonal evolution with gain of additional chromosome abnormalities, most commonly involving chromosome 19 and 20, is often seen in these cases and associated with a poor outcome (median OS of 2.24 months) [4].

Establishing erythroid lineage in acute leukemia can be challenging, particularly when there is lack of morphologic evidence of erythroid maturation and since malignant erythroid blasts often lack commonly applied lineage-defining markers such as hemoglobin and glycophorin A [1]. A panel of immunohistochemical stains and various markers by flow cytometry are required for evaluation, often relying on excluding other lineages. CD36 is often expressed in erythroid blasts, such as was seen in our patient; however, this marker is not specific [1]. Potentially helpful markers include glycophorin A, hemoglobin, E-Cadherin, CD117, and CD71, although the latter two are not specific for erythroid lineage. Newer markers such as GATA1 and alpha-hemoglobin-stabilizing protein may be useful in this setting but are not routinely available in most laboratories. Additionally, GATA1 can also stain megakaryocytic lineage cells [1, 18, 19]. Alphahemoglobin-stabilizing protein has been reported to be specific and restricted to erythroid lineage [18].

MLN-FGFR1 neoplasms are aggressive and challenging to treat. These neoplasms are characterized by poor prognosis with median survival of less than 12 months without transplant and only marginally better at 24 months with transplant. If the patient is transplant eligible, intensive induction chemotherapy followed by hematopoietic stem cell transplant (HSCT) is recommended. If the patient is not eligible for induction chemotherapy or transplant, treatment options are limited. There is no established tyrosine kinase inhibitor (TKI) therapy for MLN-FGFR1 and it does not respond to imatinib $[1,2,8]$. Ponatinib, a pan-BCR-ABL tyrosine kinase inhibitor, has demonstrated in vitro activity against $F G F R 1$ among other kinases, and some data suggest efficacy in patients [20-23]. FGFR1 inhibitors such as pemigatinib are currently being studied [24] and have shown efficacy. In the absence of an available TKI therapy, allogeneic HSCT may be a potential curative option [11, 22, 25-27].

Our case highlights the importance of recognizing this rare type of transformation to PEL/EBS from an underlying chronic MLN-FGFR1. PEL/EBS transformation from a preexisting chronic myeloid neoplasm and presentation as an isolated pleural effusion is exceedingly rare. Such cases are a diagnostic challenge given the difficulty in establishing erythroid lineage and require a comprehensive workup including flow cytometry (if fresh tissue is available) and a panel of immunohistochemical stains to arrive at the diagnosis.

Data Availability Not applicable.

\section{Compliance with ethical standards}

Disclosures The authors have nothing to disclose.

Conflict of interest The authors declare that they have no conflict of interest.

Code availability Not applicable.

\section{References}

1. Swerdlow SH, Campo E, Harris NL, Jaffe ES, Pileri SA, Stein H, Thiele J, Arber DA, Hasserjian RP, Le Beau MM, Orazi A, Siebert R (2017) WHO classification of tumours of haematopoietic and lymphoid tissues. World Health Organization classification of Tumours, revised 4th edition edn. International Agency for Research on Cancer, Lyon, France

2. Foucar K, Chabot-Richards D, Czuchlewski DR, Karner KH, Reichard KK, Vasef MA, Wilson CS, Zhang Q, Culbreath K (2018) Diagnostic pathology: blood and bone marrow, 2nd edn. Elsevier, Philadelphia 
3. Reinig EF, Greipp PT, Chiu A, Howard MT, Reichard KK (2018) De novo pure erythroid leukemia: refining the clinicopathologic and cytogenetic characteristics of a rare entity. Mod Pathol 31(5): 705-717. https://doi.org/10.1038/modpathol.2017.175

4. Li H, Hasserjian RP, Kroft SH, Harrington AM, Wheaton SE, Pildain A, Ewalt MD, Gratzinger D, Hosking P, Olteanu H (2016) Pure erythroid leukemia and erythroblastic sarcoma evolving from chronic myeloid neoplasms. Am J Clin Pathol 145(4): 538-551. https://doi.org/10.1093/ajcp/aqw033

5. Dewald GW, Broderick DJ, Tom WW, Hagstrom JE, Pierre RV (1985) The efficacy of direct, 24-hour culture, and mitotic synchronization methods for cytogenetic analysis of bone marrow in neoplastic hematologic disorders. Cancer Genet Cytogenet 18(1):1-10. https://doi.org/10.1016/0165-4608(85)90032-9

6. Brockman SR, Paternoster SF, Ketterling RP, Dewald GW (2003) New highly sensitive fluorescence in situ hybridization method to detect PML/RARA fusion in acute promyelocytic leukemia. Cancer Genet Cytogenet 145(2):144-151. https://oi.org/10.1016/ s0165-4608(03)00061-x

7. Chang K, Liu JH, Yu SC, Lin CW (2018) FGFR1 translocation with concurrent myeloproliferative neoplasm, systemic mastocytosis, and lymphoblastic lymphoma: a case report. Hum Pathol 74:114-121. https://doi.org/10.1016/j.humpath.2017.10.019

8. Vega F, Medeiros LJ, Bueso-Ramos CE, Arboleda P, Miranda RN (2015) Hematolymphoid neoplasms associated with rearrangements of PDGFRA, PDGFRB, and FGFR1. Am J Clin Pathol 144(3):377-392. https://doi.org/10.1309/AJCPMORR5Z2IKCEM

9. Montenegro-Garreaud X, Miranda RN, Reynolds A, Tang G, Wang SA, Yabe M, Wang W, Fang L, Bueso-Ramos CE, Lin P, Medeiros LJ, Lu X (2017) Myeloproliferative neoplasms with $\mathrm{t}(8 ; 22)(\mathrm{p} 11.2 ; \mathrm{q} 11.2) / \mathrm{BCR}-F G F R 1$ : a meta-analysis of 20 cases shows cytogenetic progression with B-lymphoid blast phase. Hum Pathol 65:147-156. https://doi.org/10.1016/j.humpath.2017. 05.008

10. Jackson CC, Medeiros LJ, Miranda RN (2010) 8p11 myeloproliferative syndrome: a review. Hum Pathol 41(4):461-476. https://doi. org/10.1016/j.humpath.2009.11.003

11. Wang W, Tang G, Kadia T, Lu X, Li Y, Huang L, MontenegroGarreaud X, Miranda RN, Wang SA (2016) Cytogenetic evolution associated with disease progression in hematopoietic neoplasms with $\mathrm{t}(8 ; 22)(\mathrm{p} 11 ; \mathrm{q} 11) / \mathrm{BCR}-\mathrm{FGFR} 1$ rearrangement. J Natl Compr Cancer Netw 14(6):708-711. https://doi.org/10.6004/jnccn.2016. 0072

12. Wakim JJ, Tirado CA, Chen W, Collins R (2011) T(8;22)/BCRFGFR1 myeloproliferative disorder presenting as B-acute lymphoblastic leukemia: report of a case treated with sorafenib and review of the literature. Leuk Res 35(9):e151-e153. https://doi.org/10. 1016/j.leukres.2011.05.013

13. Kreft A, Burg J, Fischer T, Kirkpatrick CJ (2004) Essential thrombocythemia terminating in pure erythroleukemia. Am J Hematol 77(2):140-143. https://doi.org/10.1002/ajh.20164

14. Liu W, Hasserjian RP, Hu Y, Zhang L, Miranda RN, Medeiros LJ, Wang SA (2011) Pure erythroid leukemia: a reassessment of the entity using the 2008 World Health Organization classification. Mod Pathol 24(3):375-383. https://doi.org/10.1038/modpathol. 2010.194

15. Cornfield DB (2012) Erythroblastic sarcoma, an extremely rare variant of myeloid sarcoma. Hum Pathol 43(11):2080-2083. https://doi.org/10.1016/j.humpath.2012.03.026

16. Kebe Radulovic M, Melkic E, Grcar Kuzmanov B, Cimerman D, Strojan Flezar M (2020) Erythroblastic sarcoma presenting as a unilateral pleural effusion in a patient with myelodysplastic syndrome/myeloproliferative neoplasm-case report with cytologic and histologic findings. Diagn Cytopathol 48:E18-E21. https://doi. org/10.1002/dc. 24540

17. Tomlinson B, Raess PW, Beck RC, Moore EM (2020) Erythroblastic sarcoma with rare PDGFRA rearrangement responding to tyrosine kinase inhibitor therapy. Br J Haematol 188(6):e98-e100. https://doi.org/10.1111/bjh.16379

18. Yu H, Pinkus JL, Pinkus GS (2016) Alpha-hemoglobin-stabilizing protein: an effective marker for erythroid precursors in bone marrow biopsy specimens. Appl Immunohistochem Mol Morphol 24(1):51-56. https://doi.org/10.1097/PAI.0000000000000139

19. Lee WY, Weinberg OK, Pinkus GS (2017) GATA1 is a sensitive and specific nuclear marker for erythroid and megakaryocytic lineages. Am J Clin Pathol 147(4):420-426. https://doi.org/10.1093/ ajcp/aqx018

20. Chase A, Bryant C, Score J, Cross NC (2013) Ponatinib as targeted therapy for FGFR1 fusions associated with the 8p11 myeloproliferative syndrome. Haematologica 98(1):103-106. https://doi.org/ 10.3324/haematol.2012.066407

21. Khodadoust MS, Luo B, Medeiros BC, Johnson RC, Ewalt MD, Schalkwyk AS, Bangs CD, Cherry AM, Arai S, Arber DA, Zehnder JL, Gotlib J (2016) Clinical activity of ponatinib in a patient with FGFR1-rearranged mixed-phenotype acute leukemia. Leukemia 30(4):947-950. https://doi.org/10.1038/leu.2015.136

22. Strati P, Tang G, Duose DY, Mallampati S, Luthra R, Patel KP, Hussaini M, Mirza AS, Komrokji RS, Oh S, Mascarenhas J, Najfeld V, Subbiah V, Kantarjian H, Garcia-Manero G, Verstovsek S, Daver N (2018) Myeloid/lymphoid neoplasms with FGFR1 rearrangement. Leuk Lymphoma 59(7):1672-1676. https:// doi.org/10.1080/10428194.2017.1397663

23. Kreil SAL, Bommer M, Stegelmann F, Ethell ME, Lubking A, Martin P, Eigendorff E, Hofmann W-K, Cross NCP et al (2015) Limited efficacy of Ponatinib in Myeloproliferative neoplasms associated with FGFR1 fusion genes. Blood 126:2812-2812. https:// doi.org/10.1182/blood.V126.23.2812.2812

24. Verstovsek SVAM, Rambaldi A, Gotlib JR, Mead AJ, Hochhaus A, Kiladjian J-J, Hernandez Boluda JC, Asatiani E, Lihou C et al (2018) Interim results from Fight-203, a phase 2, open-label, multicenter study evaluating the efficacy and safety of pemigatinib (INCB054828) in patients with myeloid/lymphoid neoplasms with rearrangement of fibroblast growth factor receptor 1 (FGFR1). Blood 132:690-690. https://doi.org/10.1182/blood-2018-99110388

25. Haslam K, Langabeer SE, Kelly J, Coen N, O'Connell NM, Conneally E (2012) Allogeneic hematopoietic stem cell transplantation for a BCR-FGFR1 myeloproliferative neoplasm presenting as acute lymphoblastic leukemia. Case Rep Hematol 2012: 620967-620965. https://doi.org/10.1155/2012/620967

26. Lee H, Kim M, Lim J, Kim Y, Han K, Cho BS, Kim HJ (2013) Acute myeloid leukemia associated with FGFR1 abnormalities. Int J Hematol 97(6):808-812. https://doi.org/10.1007/s12185-013$1337-5$

27. Soler G, Nusbaum S, Varet B, Macintyre EA, Vekemans M, Romana SP, Radford-Weiss I (2009) LRRFIP1, a new FGFR1 partner gene associated with $8 \mathrm{p} 11$ myeloproliferative syndrome. Leukemia 23(7):1359-1361. https://doi.org/10.1038/leu.2009.79

Publisher's note Springer Nature remains neutral with regard to jurisdictional claims in published maps and institutional affiliations. 